\title{
The INTEGRAL Narrow-Line Seyfert 1 Galaxies
}

\author{
F. Panessa* \\ Istituto di Astrofisica Spaziale e Fisica Cosmica (IASF-INAF), via del Fosso del Cavaliere 100, \\ 00133 Roma, Italy \\ E-mail: francesca.panessaliasf-roma.inaf.it
}

\section{A. De Rosa}

Istituto di Astrofisica Spaziale e Fisica Cosmica (IASF-INAF), via del Fosso del Cavaliere 100, 00133 Roma, Italy

E-mail: alessandra.derosaliasf-roma.inaf.it

L. Bassani

Istituto di Astrofisica Spaziale e Fisica Cosmica (IASF-INAF), Via P. Gobetti 101, 40129

Bologna, Italy

E-mail: bassani@bo.iasf.cnr.it

\section{A. Bazzano}

Istituto di Astrofisica Spaziale e Fisica Cosmica (IASF-INAF), via del Fosso del Cavaliere 100, 00133 Roma, Italy

E-mail: angela.bazzanodiasf-roma.inaf.it

\section{A. Bird}

School of Physics and Astronomy, University of Southampton, Southampton, SO17 1BJ, UK

E-mail: ajbeastro.soton.ac.uk

\section{R. Landi}

Istituto di Astrofisica Spaziale e Fisica Cosmica (IASF-INAF), Via P. Gobetti 101, 40129

Bologna, Italy

E-mail: landi@iasfbo.inaf.it

\section{A. Malizia}

Istituto di Astrofisica Spaziale e Fisica Cosmica (IASF-INAF), Via P. Gobetti 101, 40129

Bologna, Italy

E-mail: maliziadiasfbo.inaf.it

\section{G. Miniutti}

Centro de Astrobiologia (CSIC-INTA), PO Box 78, 28691, Villanueva de la Cañada, Madrid, Spain

E-mail: gminiuttiecab.inta-csic.es

\section{Molina}

Istituto di Astrofisica Spaziale e Fisica Cosmica (IASF-INAF), Via Bassini 15, 20122 Milano, Italy

E-mail: molinaliasf-milano.inaf.it

\section{P. Ubertini}




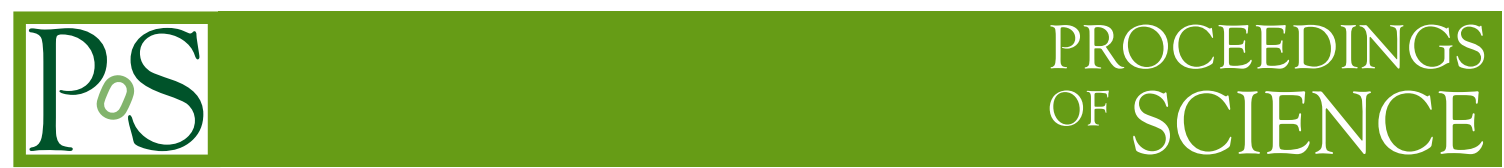

Istituto di Astrofisica Spaziale e Fisica Cosmica (IASF-INAF), via del Fosso del Cavaliere 100, 00133 Roma, Italy

E-mail: pietro.ubertini@iasf-roma.inaf.it

Narrow Line Seyfert 1 galaxies are among the most interesting class of active nuclei. Their observational properties suggest that they are high accretion rate systems probably associated with relatively small black hole masses compared to classical Seyfert 1 galaxies. However, the number of known NLSy1 detected above $10 \mathrm{keV}$ is small notwithstanding the importance of the high energy data to discriminate between the major competig models. Here we present a detailed study of a sample of 14 NLSy1 detected above $10 \mathrm{keV}$ by INTEGRAL/IBIS, through an accurate broad-band analysis using INTEGRAL data combined with XMM-Newton and Swift/XRT data. The high energy spectral properties of our sample have been related to their accretion parameters in order to unveil the nature of these extreme AGN. We have estimated the fraction of NLSy1 in the hard X-ray sky to be nearly $15 \%$, in agreement with the estimate derived from optically selected NLSy1 samples.

Narrow-Line Seyfert 1 Galaxies and their place in the Universe - NLS1, April 04-06, 2011

Milan Italy

${ }^{*}$ Speaker. 


\section{Introduction}

Narrow Line Seyfert 1 (NLSy1) are AGN with optical spectral properties similar to those of Seyfert 1 galaxies (Broad Line Seyfert 1, BLSy1), except for having the narrowest Balmer lines and strongest optical FeII lines [21]. The classical definition of NLSy1 [11] relies on three simple criteria: (i) the full width half maximum (FWHM) of the $\mathrm{H}_{\beta}$ line $<2000 \mathrm{~km} \mathrm{~s}^{-1}$; (ii) the [OIII] $\lambda 5007 / \mathrm{H}_{\beta}<3$; and (iii) unusually strong FeII.

NLSy1 show their extreme characteristics at X-rays [8], i.e., displaying strong variability and a prominent soft excess emission below $\sim 1 \mathrm{keV}$ ([9], [5], [23]). The 2-10 keV spectral slope is usually steeper in NLSy1 ([15], [6]) and a sharp spectral drop at about $7 \mathrm{keV}$ has been observed in some objects ([25], [16], [4]).

A possible interpretation for the peculiar observational properties of NLSy1 is that these systems are accreting close to their Eddington limit, suggesting that, compared to their BLSy1 counterparts, they should host smaller black hole masses, as measured in the majority of the sources ([26], [12]).

NLSy1 have been poorly investigated at hard X-rays (above $10 \mathrm{keV}$ ). Recent hard X-ray surveys performed by the IBIS instrument [24] on board INTEGRAL [27] and the BAT instrument [1] on board Swift (Geherels et al. 2004) are imaging the hard X-ray sky providing a number of sources detected for the first time at these energies with half of them newly discovered ([17], [14], [22]).

More than 700 hard X-ray sources have been detected over the whole sky above $20 \mathrm{keV}$ in the fourth IBIS survey [3], down to an average flux level of about $1 \mathrm{mCrab}$ and with positional accuracies ranging between 0.2 and $\sim 5$ arcmin. Among the AGN detected by IBIS, a small sample of NLSy1 is identified. A first analysis of the hard X-ray properties of a sample of five NLSy1 detected by IBIS has been presented in [18], which combined Swift/XRT and INTEGRAL/IBIS spectra.

\section{The sample}

Here we present the broad-band X-ray properties of a sample of 14 NLSy1 detected by INTEGRAL and present in the fourth IBIS catalogue [3]. Most of the IGR sources in the sample have been classified during the optical follow-up campaigns which are ongoing to identify the INTEGRAL IBIS sources ([19] and references therein). See the web page:

http://www.iasfbo.inaf.it/extras/IGR/main.html for the information about the INTEGRAL sources identified using optical or near-infrared (NIR) observations. New XMMNewton and Swift/XRT data have been combined with the INTEGRAL data to obtain a wide energy range up to $150 \mathrm{keV}$.

\section{The broad-band data}

The IBIS/INTEGRAL data used in this work have been collected in the 4th IBIS survey [3] consisting of 7355 pointings (called Science Windows, ScW, lasting about 2000 sec each) performed from the beginning of the Mission in November 2002 up to April 2008 and including both all available public and Core Programme data. IBIS/ISGRI images for each available pointing were 

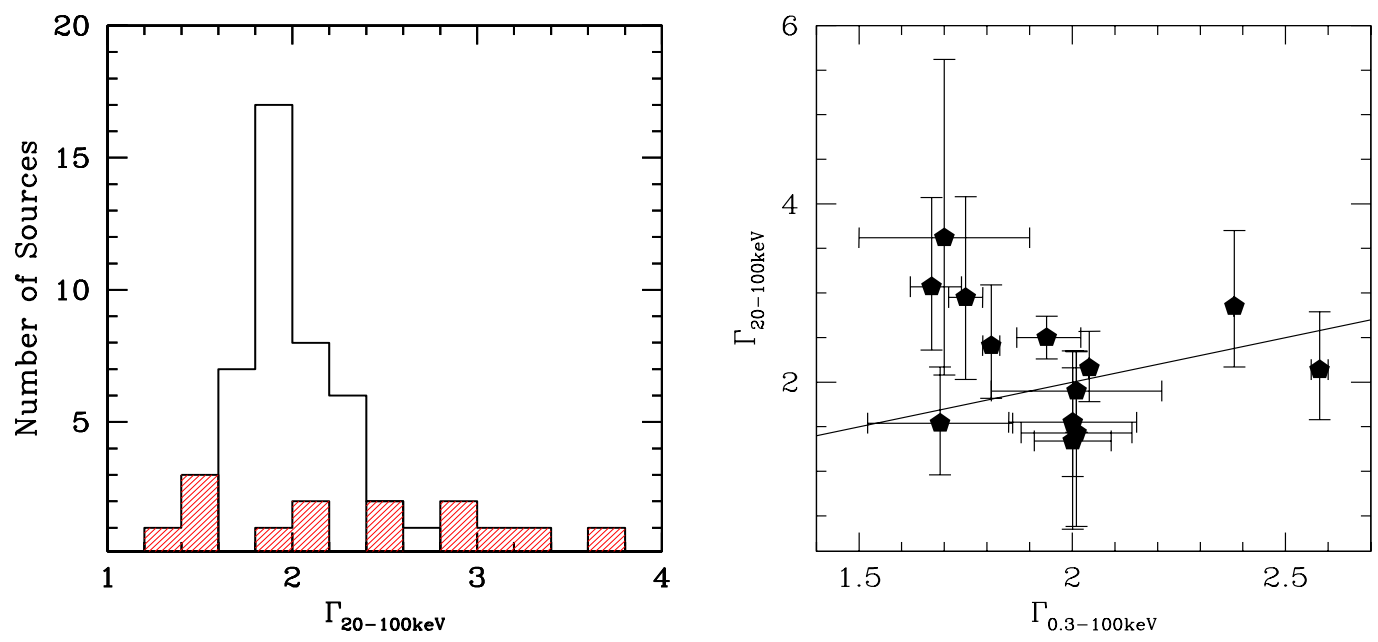

Figure 1: Left panel: Distribution of the 20-100 keV photon index for our sample of NLSy1 (shaded histogram) and a sample of hard X-ray selected BLSy1, Molina et al. (in preparation). Right panel: 20-100 $\mathrm{keV}$ photon index versus the $0.3-100 \mathrm{keV}$ broad-band photon index in NLSy1. The one-to-one relation has also been drawn.

generated in various energy bands using the ISDC off-line scientific analysis software OSA [10] version 7.0. Then all images have been mosaicked to create significance map at revolution level (each orbit lasting about 3 days) and for all the available pointings. Count rates at the position of the source were extracted from individual images in order to provide light curves in various energy bands; from these light curves, average fluxes were then extracted and combined to produce an average source spectrum (see [3] for details).

XMM-Newton/XRT and INTEGRAL spectra have been combined to obtain a $0.3-150 \mathrm{keV}$ broad-band spectrum. Since the XMM-Newton/XRT and INTEGRAL observations are not simultaneous, a cross-calibration constant $C$ is left free to vary to take into account possible crosscalibration mismatches between the two instruments but more importantly in these objects, to consider variability effects. With the aim of characterizing broadly the spectral properties of the sample, to a baseline model composed of a simple power-law with absorption fixed at the Galactic value, we have added extra components to model spectral residuals.

\section{Results}

NLS1s usually exhibit very steep spectral slopes, therefore hard-X-ray bright sources are very rare among this class. Here we present the averaged spectral properties of the first sizable sample of NLSy1 detected at hard X-rays. Hard X-ray NLSy1 look very heterogeneous in their broadband properties, displaying a broad range of hard X-ray (20-100 keV) photon indeces, distributed from quite flat $(\sim 1.3)$ to very steep $(\sim 3.6)$ slopes; at these energies no clear separation between the BLSy1 and NLSy1 slopes distributions is found, as also confirmed by a Kolmogorov-Smirnov (K-S) test (probability $P=0.030$ ). The distribution of the $20-100 \mathrm{keV}$ photon index for our sample of NLSy1 (shaded histogram) and for the BLSy1 in Molina et al. in preparation is shown in Fig- 


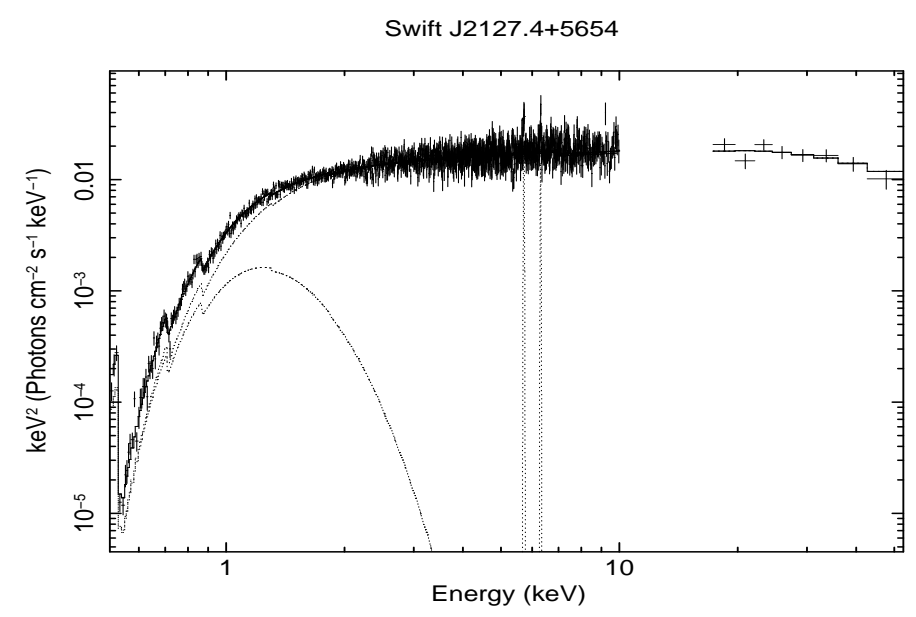

Figure 2: Broad-band XMM-Newton and INTEGRAL best-fit spectra. The continuum is described by a pexrav model, plus two gaussian narrow components and a MEKAL model for the soft X-ray emission.

ure 1. In only one source, SwiftJ2127.4+5654 (see Figure2), a high energy cut-off was constrained and a relatively low value ( $\mathrm{E}_{\text {cut }-o f f} \sim 50 \mathrm{keV}$ ) was found, confirming previous Suzaku and IBIS measurements ([20], [18]). The hard X-ray selection is not efficient in detecting strong soft X-ray NLSy1, indeed only one source (IGR J19378-0617) shows a dominant soft X-ray emission while the presence of a fully or partially covering absorption component is more frequent (in four out of ten sources analyzed in this work). With XMM-Newton data, we almost always detect the presence of an Fe line (with profiles going from moderately broad to narrow). A proper determination of the reflection strength is limited by the non simultaneity of the X-ray and hard X-ray observations, this is particularly true in this class of sources where X-ray variability (in flux and spectrum) is strong, both on short and long timescales, as here demonstrated by the XMM-Newton light curves and in those source where multiple observations were available. We have compared the INTEGRAL and BAT hard X-rays average fluxes and found that they are almost consistent, with a maximum flux variation of $\sim 30 \%$ for the most extreme source $1 \mathrm{H} 0323+342$, a blazar-like NLSy 1 already known to be variable [7].

The peculiar properties of NLSy1 have been commonly associated to the small black hole masses and relative high Eddington ratios. Black hole masses are usually estimated through massradius relations, the latter derived from the optical continuum luminosity [13], however it must be taken in mind that large uncertainties affect these estimates. The distribution of the black hole masses in our sample peaks at $\sim 10^{7} \mathrm{M}_{\odot}$, confirming the findings that NLSy1 host preferentially small black holes and possibly suggesting that the hard X-ray origin of the sample is not introducing any particular selection effect in the black hole mass range. This result is similarly found in an X-ray selected sample where [2] have shown that NLSy1 have the smallest BH masses when compared to BLSy1. On the other hand, the Eddington ratios distribution peaks at $10^{-2}$ suggesting that hard X-ray NLSy1 occupy the lower tail of Eddington ratios distribution of NLSy1.

Finally, we estimate that the fraction of NLSy1 in the hard X-ray sky is about $15 \%$ of the type 1 AGN population, in agreement with the optically selected sample fraction. 


\section{Acknowledgments}

FP acknowledges support by INTEGRAL ASI I/033/10/0 and ASI/INAF I/009/10/0. GM thanks the Spanish MICINN grant AYA2010-21490-C02-02 for support.

\section{References}

[1] S. D. Barthelmy, et al. 2005, Space Sci.Rev., 120, 143

[2] S. Bianchi, et al. 2009, A\&A, 501, 915

[3] A. J. Bird, et al. 2010, ApJS, 186, 1

[4] T. Boller, et al. 2003, MNRAS, 343, L89

[5] T. Boller, Brandt, W. N., \& Fink, H. 1996, A\&A, 305, 53

[6] W. N. Brandt, Mathur, S., \& Elvis, M. 1997, MNRAS, 285, L25

[7] L. Foschini, et al. 2009, Advances in Space Research, 43, 889

[8] L. C. Gallo, 2006, MNRAS, 368, 479

[9] I. M. George, et al. 2000, ApJ, 531, 52

[10] A. Goldwurm, et al. 2003, A\&A, 411, L223

[11] R. W. Goodrich, 1989, ApJ, 342, 224

[12] D. Grupe, \& Mathur, S. 2004, ApJL, 606, L41

[13] S. Kaspi, et al. 2000, ApJ, 533, 631

[14] R. Landi, et al. 2010, MNRAS, 403, 945

[15] K. M. Leighly, 1999, , 125, 317

[16] A. L. Longinotti, et al. 2003, A\&A, 410, 471

[17] A. Malizia, et al. 2010, MNRAS, 408, 975

[18] A. Malizia, et al. 2008, MNRAS, 389, 1360

[19] N. Masetti, et al. 2010, A\&A, 519, A96

[20] G. Miniutti, et al. 2009, MNRAS, 398, 255

[21] D. E. Osterbrock, \& Pogge, R. W. 1985, ApJ, 297, 166

[22] J. Tueller, et al. 2010, ApJS, 186, 378

[23] T. J. Turner, George, I. M., \& Netzer, H. 1999, ApJ, 526, 52

[24] P. Ubertini, et al. 2003, A\&A, 411, L131

[25] P. Uttley, et al. 2004, MNRAS, 347, 1345

[26] A. Wandel, Peterson, B. M., \& Malkan, M. A. 1999, ApJ, 526, 579

[27] C. Winkler, et al. 2003, A\&A, 411, L1 